\title{
Pigmented antibiotic production by Streptomyces coelicolor A3(2): kinetics and the influence of nutrients
}

\author{
Glyn Hobbs, ${ }^{1}$ Catherine M. Frazer, ${ }^{1}$ David C. J. Gardner, ${ }^{2}$ Fiona Flett ${ }^{2}$ \\ and STEPHEN G. Oliver ${ }^{1,2 *}$ \\ Manchester Biotechnology Centre ${ }^{1}$ and Department of Biochemistry and Applied Molecular Biology, ${ }^{2}$ \\ University of Manchester Institute of Science and Technology, PO Box 88, Manchester M60 1QD, UK
}

(Received 23 March 1990; revised 2 July 1990; accepted 17 July 1990)

\begin{abstract}
The production of the pigments actinorhodin and undecylprodigiosin by Streptomyces coelicolor A3(2) was examined in a chemically defined medium which permits dispersed growth of the organism. The physiological controls on the production of the two pigments were markedly disparate. Actinorhodin production occurred mainly in the stationary phase of batch cultures grown with glucose and sodium nitrate as the principal carbon and nitrogen sources. In the same batch cultures, undecylprodigiosin accumulated during the exponential growth phase. The production of both pigments was sensitive to the levels of ammonium and phosphate in the medium. Actinorhodin production was exquisitely sensitive to ammonium concentration, and was completely inhibited by as little as $1 \mathrm{mM}$-ammonium chloride, whereas more than $50 \mathrm{mM}$-ammonium chloride was required to prevent undecylprodigiosin production. A similar, but less extreme effect was seen with phosphate: actinorhodin production was completely inhibited by 24 mM-phosphate, whereas undecylprodigiosin was still formed at this high phosphate concentration. The effects of ammonium inhibition of pigmented antibiotic production were relieved by reducing the concentration of phosphate in the medium, but changing the ammonium concentration had no effect on phosphate inhibition. Thus the regulation of pigment production by these two nutrients is interrelated, with phosphate control being epistatic to that of ammonium. The results implicate a phosphorylated intermediate as a major regulator of secondary metabolite synthesis by $S$. coelicolor.
\end{abstract}

\section{Introduction}

The genetics of Streptomyces coelicolor A3(2) has been intensively studied using both classical and molecular approaches (Hopwood, 1988) but the physiology of this organism remains relatively poorly defined. Hodgson (1982) used both genetical and physiological techniques to investigate glucose uptake and metabolism by $S$. coelicolor. Since then, progress has been slow, probably because of the difficulties in culturing $S$. coelicolor in chemically defined growth media. We (Hobbs et al., 1989) have recently devised a medium which overcomes many of these difficulties and allows the dispersed growth of $S$. coelicolor under chemically defined conditions. The medium employs a negatively charged polymer (Junlon PW110) to obtain dispersion and permits the growth of $S$. coelicolor cultures which are both physiologically homogeneous and readily reproducible from experiment to experiment. In this paper, we report the use of this culture system to investigate the kinetics of secondary metabolite production in batch culture and to determine the effect of medium nutrient composition on product formation.

$S$. coelicolor synthesizes two chemically distinct pigments which are generally regarded as secondary metabolites: actinorhodin, a diffusible red-blue $\mathrm{pH}$ indicator, and undecylprodigiosin, a red cell-wallassociated compound (Rudd \& Hopwood, 1980). Genes which determine the synthesis of these two pigments have been identified (Rudd \& Hopwood, 1979, 1980; Feitelson et al., 1985; Malpartida \& Hopwood, 1986) and cloned (Feitelson \& Hopwood, 1983; Feitelson et al., 1986; Malpartida \& Hopwood, 1984, 1986). However, the physiological controls which operate on pigment production in $S$. coelicolor are unknown. Feitelson et al. (1985) reported that the onset of undecylprodigiosin production was delayed to mid- or late-exponential phase in liquid cultures of $S$. coelicolor grown on a complex beef extract/peptone medium (AM medium; Okanishi et al., 1974). It was not clear that exponential growth was actually achieved and the complexity of the medium precluded identification of a nutritional limitation which 
might have triggered pigment production. We have re-examined pigment production in $S$. coelicolor under defined physiological conditions. We demonstrate that the kinetics of accumulation of undecylprodigiosin and actinorhodin are markedly disparate and that the composition of the growth medium affects the synthesis of both products.

\section{Methods}

Organism. Streptomyces coelicolor A3(2) strain 1147 (Hopwood, 1959) was used. This is a prototrophic strain which contains plasmids SCP1 and SCP2.

Inoculum preparation. The inoculum used throughout originated from a single frozen spore stock culture. The spore stock was spread on a plate of sporulation agar (Hobbs et al., 1989) and incubated for 10-14 d at $30^{\circ} \mathrm{C}$ to allow sporulation. Spores were then streaked by wire loop to produce confluent growth on several plates of sporulation agar. Distilled water $(5 \mathrm{ml})$ was added to each plate and the surface gently scraped to release the spores. Suspensions were harvested by centrifugation and washed twice with distilled water. Before use as inocula, the spores were dispersed for $10 \mathrm{~min}$ in a sonic bath. Inocula were adjusted to a final concentration of $2 \times 10^{6}$ spores $\mathrm{ml}^{-1}$.

Bacterial growth and its estimation. Basal medium (HMM) was as described by Hobbs et al. (1989); Junlon PW 110 was a gift from Honeywill \& Stein Ltd (Greenfield House, 69/73 Manor Road, Wallington, Surrey, UK).

Cultures were grown in $250 \mathrm{ml}$ flasks containing $100 \mathrm{ml}$ medium at $30{ }^{\circ} \mathrm{C}$ on an orbital shaker at 200 r.p.m. Biomass concentrations were estimated from optical density measurements at $450 \mathrm{~nm}$ (Gilford 250 spectrophotometer) and their equivalence in cell dry weight was calculated as described previously (Hobbs et al., 1989).

Pigment extraction and quantification. At each time point, $10-20 \mathrm{ml}$ culture samples were taken and biomass estimated as described. The sample was then divided into two 5-10 ml aliquots for the estimation of the pigments. Actinorhodin was extracted by adding an equal volume of $1 \mathrm{M}$-sodium hydroxide to one of the culture aliquots. The sample was then centrifuged at $1100 \mathrm{~g}$ for $5 \mathrm{~min}$ and the pigment concentration determined by measuring the absorbance of the supernatant at $633 \mathrm{~nm}$ and applying the formula of Horinouchi \& Beppu (1984). Undecylprodigiosin was extracted from the cell pellet harvested by centrifugation $(1100 \mathrm{~g}$ for $5 \mathrm{~min})$ from the remaining culture aliquot. The pigment was extracted from the cell pellet using the method of Williams et al. (1956) but modified by incorporating a sonication step prior to extraction with alkali. Sonication was conducted in an MSE sonicator at full power using the medium-sized probe. Three $1 \mathrm{~min}$ 'on', $30 \mathrm{~s}$ 'off' cycles were used to obtain cell breakage. After removing cell debris by centrifugation, absorbance measurements were made at $533 \mathrm{~nm}$ and the pigment concentration calculated as described by Horinouchi \& Beppu (1984).

Glucose utilization. Glucose concentrations in the media were estimated using a glucose oxidase test kit (Sigma).

\section{Results}

\section{The nature of the inoculum}

To obtain reproducible batch growth and pigment production with $S$. coelicolor, we have devised a defined

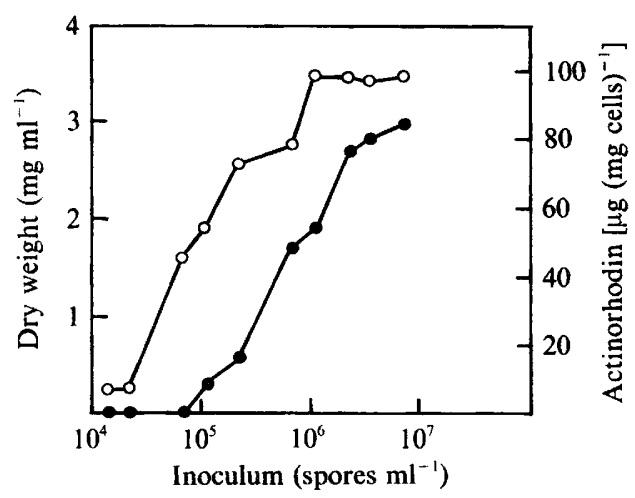

Fig. 1. Effect of inoculum size on biomass $(O)$ and actinorhodin (O) production. Biomass and actinorhodin concentrations were determined spectrophotometrically. Spore counts were made with a haemocytometer. All measurements were made in triplicate and the results presented are the means.

medium (HMM) which, through incorporation of a charged polymer, permits growth of the organism as dispersed filaments (Hobbs et al., 1989). The second major factor which influences the reproducibility of batch culture experiments with this organism is the nature of the inoculum. In all experiments reported here, we used fresh spore inocula to minimize the lag phase. All inocula were derived from a single spore stock.

The medium used in this study (HMM) was as described previously (Hobbs et al., 1989), using glucose at a concentration of $2 \mathrm{~g} \mathrm{l}^{-1}$. The effect of inoculum size on the yield of both biomass and the blue pigment, actinorhodin, was determined. Biomass concentration was determined $116 \mathrm{~h}$ after inoculation, by which time all cultures had entered the stationary phase, irrespective of the original inoculum size. Fig. 1 demonstrates that biomass yield was proportional to inoculum size at spore concentrations between $3.7 \times 10^{4}$ and $8.7 \times 10^{5}$ spores $\mathrm{ml}^{-1}$. Lack of proportionality to biomass yield with larger inocula was not investigated further, but it may have resulted from the mutual inhibition of spore germination by high spore densities, or may reflect the absolute biomass/substrate yield for this strain. Conversely, the failure of low spore concentrations to establish a growing culture may indicate that some factor which is essential for germination is leached from the spores and is present in insufficient concentration when small inocula are used. Actinorhodin, on the other hand, was only produced at inoculum sizes of $>1 \times 10^{5}$ spores $\mathrm{ml}^{-1}$ (Fig. 1). Cultures grown from small inocula tended to produce pellets rather than dispersed filaments. This response may have resulted in a significant proportion of biomass entering the stationary phase before other conditions essential to antibiotic production, either 


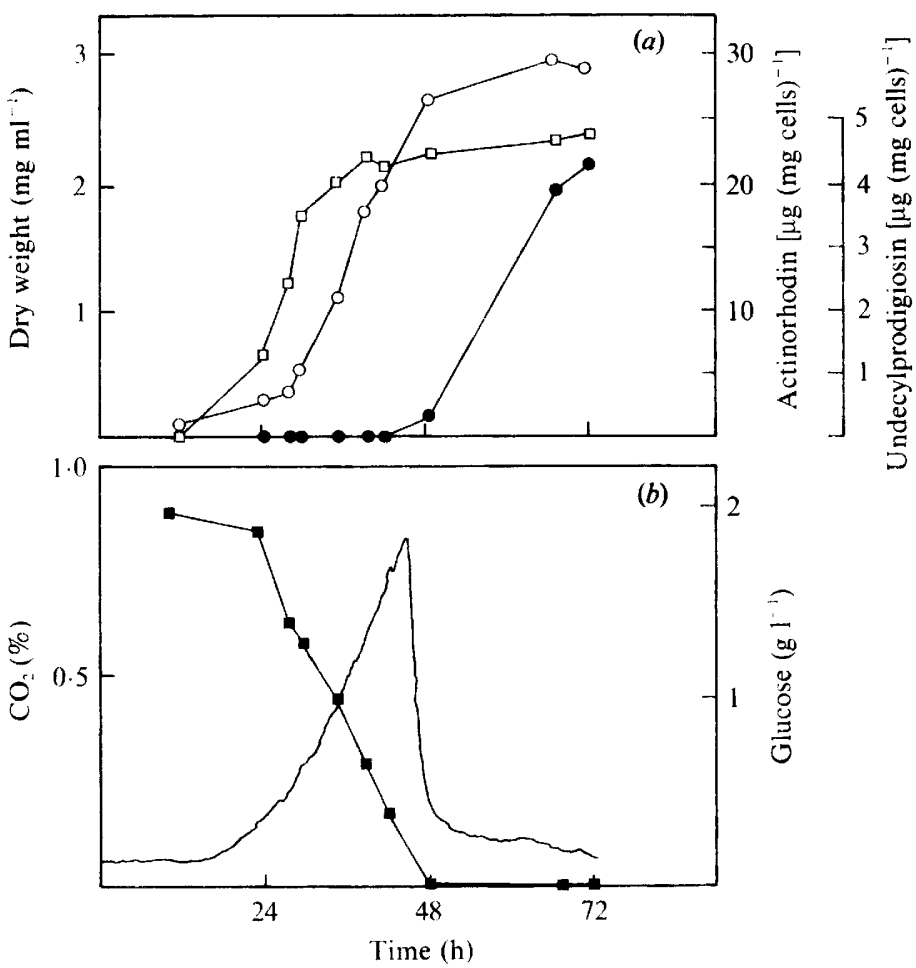

Fig. 2. (a) Production of biomass, actinorhodin and undecylprodigiosin in batch cultures grown in $250 \mathrm{ml}$ conical flasks containing $100 \mathrm{ml} \mathrm{HMM}$. Measurements were made in duplicate in two separate cultures and the results presented are the means. Biomass $(O)$, actinorhodin $(\bullet)$ and undecylprodigiosin $(\square)$ were measured spectrophotometrically. (b) Glucose concentration in the medium ( $\mathbf{(})$ and $\mathrm{CO}_{2}$ production (-).

within or outside the cells, had been satisfied. Alternatively, germinating spores may excrete products into the medium whose concentration is critical to the final pigment yield. In all subsequent experiments, an inoculum size of $2 \times 10^{6}$ spores $\mathrm{ml}^{-1}$ was employed.

\section{Kinetics of growth and antibiotic production}

The relationship between growth and pigment production was studied in batch cultures grown in HMM containing $2 \mathrm{~g}$ glucose $\mathrm{l}^{-1}$. The kinetics of biomass accumulation, measured spectrophotometrically, is shown in Fig. 2(a). The similar growth curve (until glucose was exhausted) obtained by monitoring the concentration of $\mathrm{CO}_{2}$ in the exhaust gases (Fig. 2b) supports the contention that optical density measurements reliably monitor growth in Junlon-containing media (Hobbs et al., 1989). Production of undecylprodigiosin paralleled the accumulation of biomass in the culture (Fig. 2a), indicating that the production of this pigment is growth-associated. While the kinetics of undecylprodigiosin production do not conform to those
Table 1. Effects of nitrogen sources on mean culture doubling time and on actinorhodin and undecylprodigiosin production

The medium used was HMM supplemented with amino acids or inorganic nitrogen sources at $50 \mathrm{mM}$ with respect to nitrogen. Pigment production was measured after $116 \mathrm{~h}$. Cultures were grown in duplicate and the results are means.

\begin{tabular}{lccc}
\hline \hline $\begin{array}{c}\text { Nitrogen } \\
\text { source }\end{array}$ & $\begin{array}{c}\text { Doubling } \\
\text { time (h) }\end{array}$ & $\begin{array}{c}\text { Actinorhodin } \\
{\left[\mu \mathrm{g}(\mathrm{mg} \text { cells })^{-1}\right]}\end{array}$ & $\begin{array}{c}\text { Undecylprodigiosin } \\
{\left[\mu \mathrm{g}(\mathrm{mg} \text { cells })^{-1}\right]}\end{array}$ \\
\hline $\begin{array}{c}\text { Ammonium } \\
\text { chloride }\end{array}$ & 7.3 & 0 & 5 \\
Leucine & 7.8 & 0 & 18 \\
Proline & $9 \cdot 3$ & 46 & 39 \\
$\begin{array}{l}\text { Glycine } \\
\text { Glutamine }\end{array}$ & $8 \cdot 5$ & 0 & 35 \\
Sodium & 7.6 & 0 & 19 \\
nitrate & 13.4 & 50 & 60 \\
Ammonium & & & 25 \\
nitrate & 7.5 & 0 & \\
\hline \hline
\end{tabular}

usually displayed by a secondary metabolite, neither can this red pigment be considered a product of primary metabolism. In continuous culture, the titre of undecylprodigiosin in the steady state is inversely proportional to dilution rate (our unpublished results). In contrast, actinorhodin is produced during the period of growth cessation (Fig. 2a) and may therefore be regarded as more typical of a secondary metabolite, since such compounds are normally produced under sub-optimal growth conditions (Rose, 1979).

\section{Effect of nitrogen source on actinorhodin production}

HMM contains sodium nitrate as the sole nitrogen source (Hobbs et al., 1989). To investigate whether actinorhodin production is subject to nitrogen catabolite inhibition we grew $S$. coelicolor on a variety of nitrogen sources with glucose always forming the principal source of carbon. Of the nitrogen sources studied, only sodium nitrate and proline permitted the production of actinorhodin (Table 1). No pigment was produced with ammonium chloride or ammonium nitrate. $S$. coelicolor grew more rapidly with ammonia than with nitrate or proline as the sole nitrogen source (Table 1). These data might indicate that actinorhodin production is related to growth. However, the growth rate on glycine differed by only $10 \%$ from that on proline (Table 1) and no actinorhodin was produced in glycine-grown cultures. Moreover, data from other systems (e.g. Bossinger et al., 1974) have demonstrated that growth rate is unrelated to the severity of repression which nitrogen exerts on sensitive metabolic pathways. To examine further the nature of such inhibitory effects in $S$. coelicolor, we 


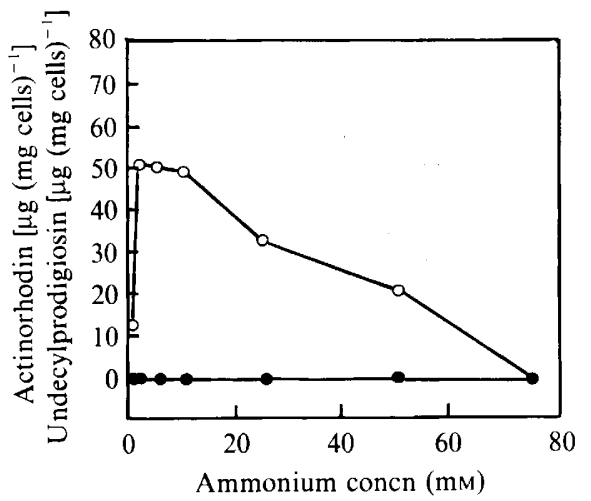

Fig. 3. Effect of ammonium concentration on the production of actinorhodin (๑) and undecylprodigiosin $(O)$ in the presence of $11 \mathrm{mM}$-phosphate. The medium used was HMM supplemented with various concentrations of ammonium chloride as the sole nitrogen source. Cultures were grown in $250 \mathrm{ml}$ conical flasks containing $100 \mathrm{ml}$ medium. Pigments were measured after $72 \mathrm{~h}$ of growth. Determinations were performed on duplicate cultures and the results are the means.

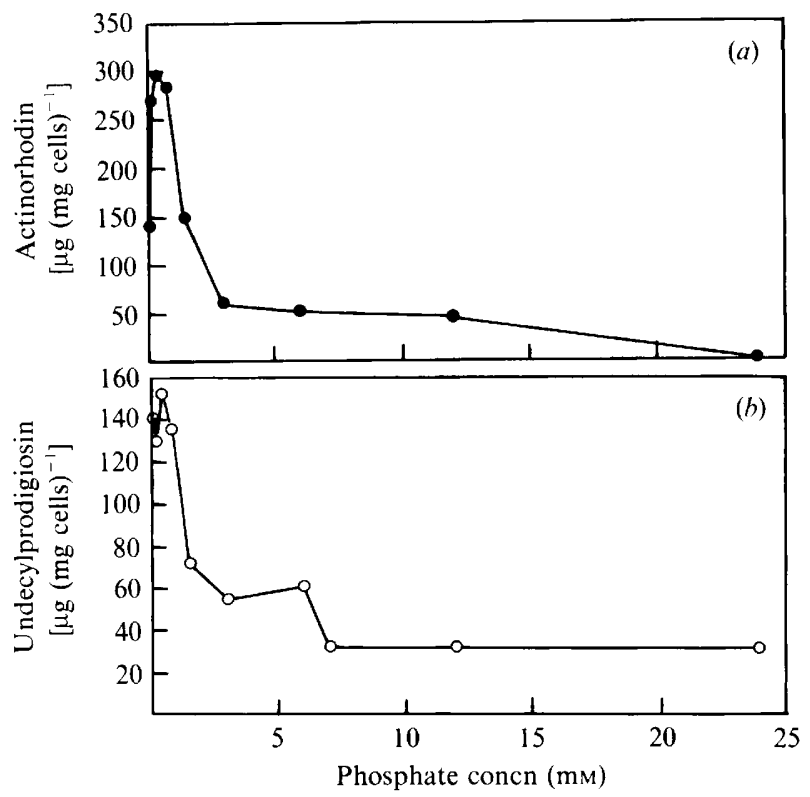

Fig. 4. Effect of phosphate concentration on the production of actinorhodin $(\bullet)$ and undecylprodigiosin $(O)$. The medium was HMM supplemented with a range of phosphate concentrations. Pigments were measured after $72 \mathrm{~h}$ and determinations performed on duplicate cultures; the results are the means.

investigated the impact of ammonium on the production kinetics of both actinorhodin and undecylprodigiosin.

\section{Ammonium inhibition of pigment production}

S. coelicolor was grown in media containing a range of concentrations of ammonium chloride as sole nitrogen

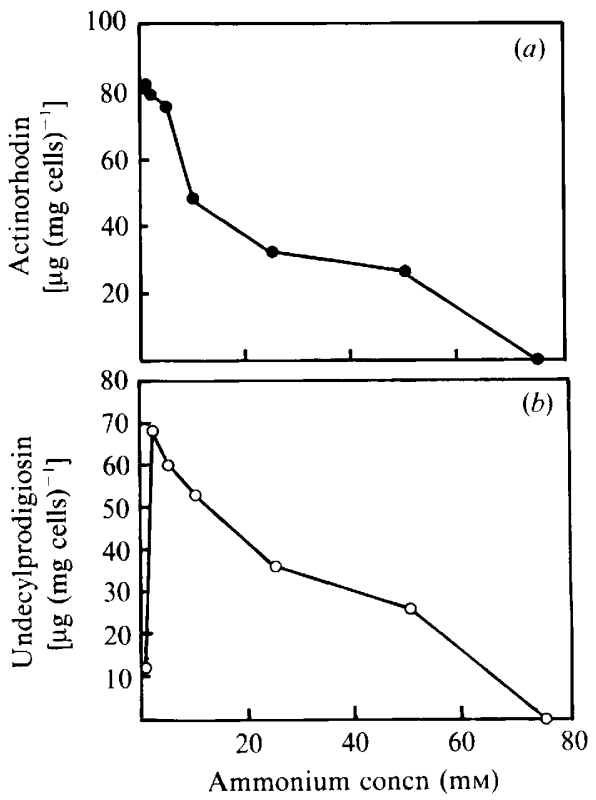

Fig. 5. Effect of ammonium concentration on the production of actinorhodin (๑) and undecylprodigiosin $(O)$ in HMM containing $1 \mathrm{~mm}$-phosphate. Cultures were grown in $250 \mathrm{ml}$ conical flasks containing $100 \mathrm{ml}$ medium. Pigments were measured after $72 \mathrm{~h}$. Determinations were performed on duplicate cultures and the results are the means.

source. The data (Fig. 3) indicate that actinorhodin production is exquisitely sensitive to inhibition by ammonium, whereas undecylprodigiosin is not sensitive below an ammonium concentration of $50 \mathrm{~mm}$. Similar data were obtained with ammonium nitrate.

\section{Effect of phosphate concentration on pigment production}

Phosphate is a major factor in the synthesis of a wide range of antibiotics (Martín, 1977) and it has been suggested that phosphorylated metabolites are important control elements. Accordingly, we examined the effect of phosphate concentration on pigment production by $S$. coelicolor in HMM with nitrate as sole nitrogen source (Fig. 4). Phosphate concentrations greater than $24 \mathrm{~mm}$ completely inhibited actinorhodin production and the yield of actinorhodin increased with decreasing phosphate concentration, to an optimum at $0.38 \mathrm{~mm}$. The optimum phosphate concentration for the production of the two pigments was $1 \mathrm{mM}$. Higher concentrations of phosphate also inhibited undecylprodigiosin synthesis but, in contrast to actinorhodin, did not completely prevent it. Since phosphate inhibited pigment production even on nitrate, a permissive nitrogen source, we examined next the interrelationship between inhibition of pigment production by phosphate and by ammonium. 
Low phosphate relieves the ammonium repression of pigment production

At the phosphate concentration in HMM (11 mM), ammonium inhibited production of actinorhodin at concentrations below $1 \mathrm{mM}$. In contrast, undecylprodigiosin formation was fully inhibited only at ammonium concentrations above $75 \mathrm{mM}$. Reducing the phosphate concentration in HMM to $1 \mathrm{mM}$ had no effect on the inhibition of undecylprodigiosin production by high concentrations of ammonium (compare Figs 3 and 5), but it had a marked effect on actinorhodin production, in that the concentration of ammonium required for complete inhibition was increased from $1 \mathrm{mM}$ to more than $50 \mathrm{mM}$ (Fig. 5). This indicated that secondary metabolism in $S$. coelicolor is impeded by both ammonium and phosphate and that there is some interrelationship between these two control systems.

\section{Discussion}

The results demonstrate that the two pigmented antibiotics synthesized by $S$. coelicolor are differently controlled. Actinorhodin is a secondary metabolite, the production of which is exquisitely sensitive to inhibition or repression by ammonium and is also prevented by high concentrations of phosphate. In the culture conditions employed in our experiments, undecylprodigiosin is produced during growth. Although it is evident, both from batch culture experiments in rich media (Feitelson et al., 1985) and from our own unpublished data from continuous cultures, that this red pigment is not a growth-linked primary product, physiological regulation of its production differs markedly from that of actinorhodin. Its synthesis is only moderately affected by ammonium or phosphate concentration whereas synthesis of actinorhodin is severely inhibited by both these nutrients. The chemically related pigment prodigiosin, produced by Serratia marcescens, is also sensitive to levels of phosphate in the medium (Witney et al. 1977). The site of phosphate inhibition, in this instance, was believed to be enzymes forming the precursors of prodigiosin. We provide no evidence for such a mechanism in $S$. coelicolor. However, a direct interaction with biosynthetic enzymes might explain the differential sensitivity of undecylprodigiosin and actinorhodin to phosphate.

We suggest that ammonium and phosphate are both major controllers of secondary metabolism in $S$. coelicolor A3(2) and that their control systems are interrelated in some way. A reduction in the phosphate concentration is able to relieve ammonium inhibition of actinorhodin production (Fig. 5). The converse is not true: inhibition of actinorhodin production by high levels of phosphate is not relieved by reducing the concentration of ammonium.

This asymmetry in the relationship between ammonium and phosphate control of actinorhodin production implicates a phosphorylated intermediate as a major regulatory element for secondary product synthesis. Martín (1977) summarized the possible candidates: ATP (Silaeva et al., 1965), adenylate energy charge (Atkinson, 1969), polyphosphates (Harold, 1966) or highly phosphorylated nucleotides. Ochi (1987) emphasized the importance of the level of GTP and its hyperphosphorylated derivative, ppGpp (Gallant, 1979), in controlling synthesis of A-factor, and thereby streptomycin production (Hara \& Beppu, 1982), in Streptomyces griseus. Our own studies throw no light on the nature of a phosphorylated regulatory molecule in $S$. coelicolor; however, recent work by Bibb \& Strauch (1990) appears to rule out ppGpp as a candidate. The facility with which both the genetics and the physiology of $S$. coelicolor may now be controlled suggests that further investigations should definitively answer the question of how secondary metabolism is regulated in this organism.

This work was supported by the 'Antibiotics and Recombinant DNA' initiative, which is sponsored by the SERC Biotechnology Directorate, the Department of Trade and Industry, Beecham Pharmaceuticals, Celltech, Glaxo Group Research and ICI Pharmaceuticals. We are grateful to John Cullum, Paul Broda and, especially, Iain Hunter for many useful discussions.

\section{References}

AtKinson, D. E. (1969). Regulation of enzyme function. Annual Review of Microbiology 23, 47-68.

BiBb, M. \& STRAUCH, E. (1990). The stringent response in Streptomyces coelicolor A3(2). Journal of Cellular Biochemistry 14A, 86.

Bossinger, J., LaWther, R. P. \& COOPER, T. G. (1974). Nitrogen repression of allantoin degradative enzymes in Saccharomyces cerevisiae. Journal of Bacteriology 118, 821-829.

Feitelson, J. S. \& Hopwood, D. A. (1983). Cloning of a Streptomyces gene for an $o$-methyltransferase involved in antibiotic biosynthesis. Molecular and General Genetics 190, 394-398.

Feitelson, J. S., Malpartida, F. \& Hopwood, D. A. (1985). Genetic and biochemical characterization of the red gene cluster of Streptomyces coelicolor A3(2). Journal of General Microbiology 131, 2431-2441.

Feitelson, J. S., Sinha, A. M. \& Coco, E. A. (1986). Molecular genetics of red biosynthesis in Streptomyces. Journal of Natural Products 49, 988-994.

Gallant, J. A. (1979). Stringent control in Escherichia coli. Annual Review of Genetics 13, 393-415.

HAROLD, F. M. (1966). Inorganic polyphosphates in biology; structure, metabolism and function. Bacteriological Reviews 30, 772-794.

HARA, O. \& BePPU, T. (1982). Mutants blocked in streptomycin production in Streptomyces griseus - the role of a-factor. Journal of Antibiotics 35, 349-358.

Hobrs, G., Frazer, C. M., Gardner, D. C. J., Cullum, J. A. \& OlIVER, S. G. (1989). Dispersed growth of Streptomyces in liquid culture. Applied Microbiology and Biotechnology 31, 272-277. 
Hodgson, D. A. (1982). Glucose repression of carbon source uptake and metabolism in Streptomyces coelicolor A3(2) and its perturbation in mutants resistant to 2-deoxyglucose. Journal of General Microbiology 128, 2417-2430.

HoPwOOD, D. A. (1959). Linkage and the mechanism of recombination in Streptomyces coelicolor. Annals of the New York Academy of Sciences 81, 887-898.

HoPwOOD, D. A. (1988). Towards an understanding of gene switching in Streptomyces, the basis of sporulation and antibiotic production. Proceedings of the Royal Society B235, 121-138.

HoRINOUCHI, S. \& BePPU, T. (1984). Production in large quantities of actinorhodin and undecylprodigiosin induced by $a f_{S} B$ in Streptomyces lividans. Agricultural and Biological Chemistry 48, 2131-2133.

Malpartida, F. \& Hopwood, D. A. (1984). Molecular cloning of the whole biosynthetic pathway of a Streptomyces antibiotic and its expression in a heterologous host. Nature, London 309, 462-464.

Malpartida, F. \& Hopwood, D. A. (1986). Physical and genetic characterization of the gene for the antibiotic actinorhodin in Streptomyces coelicolor A3(2). Molecular and General Genetics 205, $66-73$.

Martin, J. F. (1977). Control of antibiotic synthesis by phosphate. Advances in Biochemical Engineering 6, 105-127.

OCHI, K. (1987). Metabolic initiation of differentiation and secondary metabolism by Streptomyces griseus: significance of the stringent response (ppGpp) and GTP content in relation to A-factor. Journal of Bacteriology 169, 3608-3616.

OKanishi, M., Suzuki, K. \& Umezawa, H. (1974). Formation and reversion of streptomycete protoplasts: cultural condition and morphological study. Journal of General Microbiology 80, 389-400.

Rose, A. H. (1979). Production and industrial importance of secondary products of metabolism. In Economic Microbiology, vol. 3, pp. 1-33. Edited by A. H. Rose. London: Academic Press.

RuDD, B. A. M. \& HoPwood, D. A. (1979). Genetics of actinorhodin biosynthesis by Streptomyces coelicolor A3(2). Journal of General Microbiology 114, 35-43.

RudD, B. A. M. \& HoPwood, D. A. (1980). A pigmented mycelial antibiotic in Streptomyces coelicolor: control by a chromosomal gene cluster. Journal of General Microbiology 119, 333-340.

Silaeva, S. A., Glazer, V. M., Shestakov, S. V. \& Prokofiev, M. A. (1965). Nucleotides of Bacillus brevis GB cells producing and not producing gramicidin S. Biokhimya 30, 947-955.

Williams, R. P., Green, J. A. \& RAPpoport, D. A. (1956). Studies on pigmentation of Serratia marcescens. 1. Spectral and paper chroma. tographic properties of prodigiosin. Journal of Bacteriology $7 \mathbf{i}$, $115-120$.

Witney, F. R., Failla, M. L. \& Weinberg, E. D. (1977). Phosphate inhibition of secondary metabolism in Serratia marcescens. Applied and Environmental Microbiology 33, 1042-1046. 